\title{
Design of a Locator for Designing the Shortest Incision for a Polygonal Dural Window
}

\author{
Mutsumi NAGAI, ${ }^{1}$ Naoki KAnEKO, ${ }^{1}$ Fumihiro ARAI, ${ }^{1}$ Gen KuSAKA, ${ }^{2}$ \\ and Mami ISHIKAWA ${ }^{3}$ \\ ${ }^{1}$ Department of Neurosurgery, Sano Kousei General Hospital, \\ Sano, Tochigi, Japan; \\ ${ }^{2}$ Department of Neurosurgery, Saitama Medical Center Jichi Medical \\ University, Saitama, Saitama, Japan; \\ ${ }^{3}$ Department of Neurosurgery, Edogawa Hospital, Tokyo, Japan
}

\begin{abstract}
When a wide polygonal dural window is created, a short dural incision length is preferred by surgeons because suturing a wastefully long incision line during closure is troublesome. A locator to facilitate making the shortest dural incision when creating a polygonal dural window would be helpful. We geometrically analyzed the shortest incision design for a pentagonal dural window and produced a simple locator for intraoperatively implementing this design. The design for a pentagonal dural window with the shortest incision is the same as the design for a minimum Steiner tree (MST) problem with five vertices. The MST consists of three interconnected Steiner points (SPs) with three equal, radiating branches. We produced a template of the features of the MST for a polygon (MST template) as a locator. The MST template consists of several uniform Steiner units (SUs), each of which has an SP at the center and three wings that branch off of the SP, and each SU also has three slits through which the wings of another unit can pass. This mechanism allows us to freely adjust the distance between the SPs of separate SUs. In clinical practice, we can create the shortest incision design for a quadrilateral or pentagon by arranging MST templates combining two or three SUs. If we open a wide dural window, the total incision lengths created using our method are 1-5 cm shorter than conventional incisions. The MST template accurately and easily reveals the shortest incision design.
\end{abstract}

Key words: dural incision, geometrical analysis, shortest, steiner tree, template

\section{Introduction}

In a craniotomy, a polygonal window is typically opened in the dura mater. When a wide dural window is created, a short dural incision length is preferred by surgeons because suturing a wastefully long incision line during closure is troublesome. If the dura mater is opened in the shape of a polygon, the dura mater must be incised along the line of a design that connects each vertex. Thus, we want to identify the shortest set of line segments interconnecting each vertex of a given polygon. Identifying the shortest network to connect every vertex of a polygon describes a Steiner tree problem. Thus, the solution is called a minimum

Received May 6, 2017; Accepted June 27, 2017

Copyright $\odot 2017$ by The Japan Neurosurgical Society This work is licensed under a Creative Commons AttributionNonCommercial-NoDerivatives International License.
Steiner tree (MST). Previously, we presented the shortest incision design and the method to identify it in the case of opening a quadrilateral dural window, which was a MST connecting each vertices of the quadrilateral. The MST for a given quadrilateral is a network with two three-pronged intersections (TPIs) that have equal angles $(2 / 3 \pi)$ between any two line segments, called Steiner points (SPs) (Fig. 1, left). ${ }^{1)}$

Now, there are two remaining issues to address. The first issue is that in some cases, a pentagonal dural window must be opened if a wider surgical opening is required, but the shortest incision design for a pentagonal dural window cannot be identified with the same method used for a quadrilateral window. We explored the shortest incision design for a pentagonal dural window with the other geometrical approach than that for a quadrilateral one.

The other issue is that using the plotting method of identifying the shortest design requires triangles to be drawn outside of the craniotomy to construct 
the shortest incision design, even for a quadrilateral window. ${ }^{11}$ Ideally, these triangles should be plotted on the same plane as the operative field for precise construction, but this approach cannot be applied intraoperatively because the operative field is hemispheric, not a plane. Plotting on an incorrect plane causes small error in the design. We developed a locator that can create the shortest incision design intraoperatively without requiring construction outside of the craniotomy. In this paper, we present a new template that generates the design for both quadrilateral and pentagonal dural windows.

\section{Materials and Methods}

\section{Geometrical analysis}

We want to identify the shortest set of line segments interconnecting five vertices of a given pentagon.
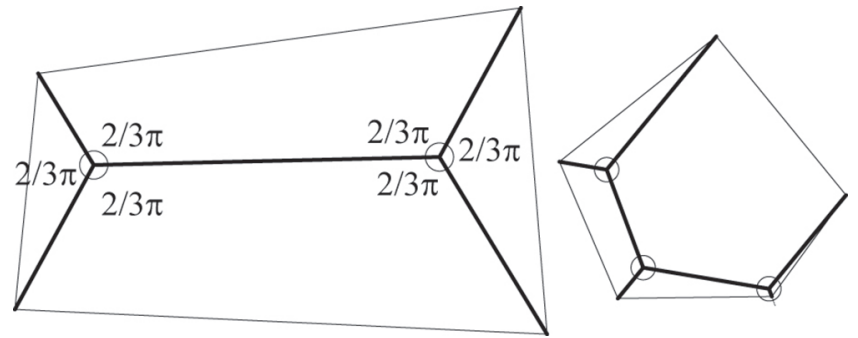

Fig. 1 The shortest incision design for a polygonal dural window. Left: The thick line is an MST for four vertices, which is the shortest network of this quadrilateral. This design has two SPs (circle) with three equally radiating branches. Right: The shortest incision design for a pentagonal dural window is shown. This design consists of three SPs and seven branches. Each angle between any two branches at the $S P$ is $2 / 3 \pi$.
The shortest incision design is the solution for an MST problem that involves five vertices. In general, the MST problem is difficult to solve if the number of vertices is large, but if the number is small, the problem is typically solved by applying Melzak's algorithm. ${ }^{2)}$ We analyzed the shortest incision design to open a pentagonal dural window by applying Melzak's algorithm.

\section{A locator for the shortest incision design (MST template)}

According to the geometrical analysis, the shortest incision design is a Steiner tree connecting all the vertices of a polygon. If a unit has an SP at the center and three equal, radiating $(2 / 3 \pi)$ branches, the combination of these components assembled by connecting each branch in a straight line creates a Steiner tree. If the distance between the two SPs of each unit is adjustable, the combination can be used as a Steiner tree locator for any polygon (MST template). We constructed such a template out of a polypropylene sheet. Each unit of this tool, called a Steiner unit (SU), has an SP at the center and three wings indicating its three branches; it also has three slits through which the wing of another SU can pass. The branch of an SU can connect with the wing of another SU by passing its wing through the slit of another SU; in this way, each SU can be smoothly repositioned to create a new combination. We can adjust the distance between the SPs freely using this mechanism (Fig. 2). In clinical practice, we created the shortest incision design by using MST template on seven cases of pentagonal cranial window. We compared the distance of the total incision line with that of conventional incision design for the same pentagon by simulating in the representative case.
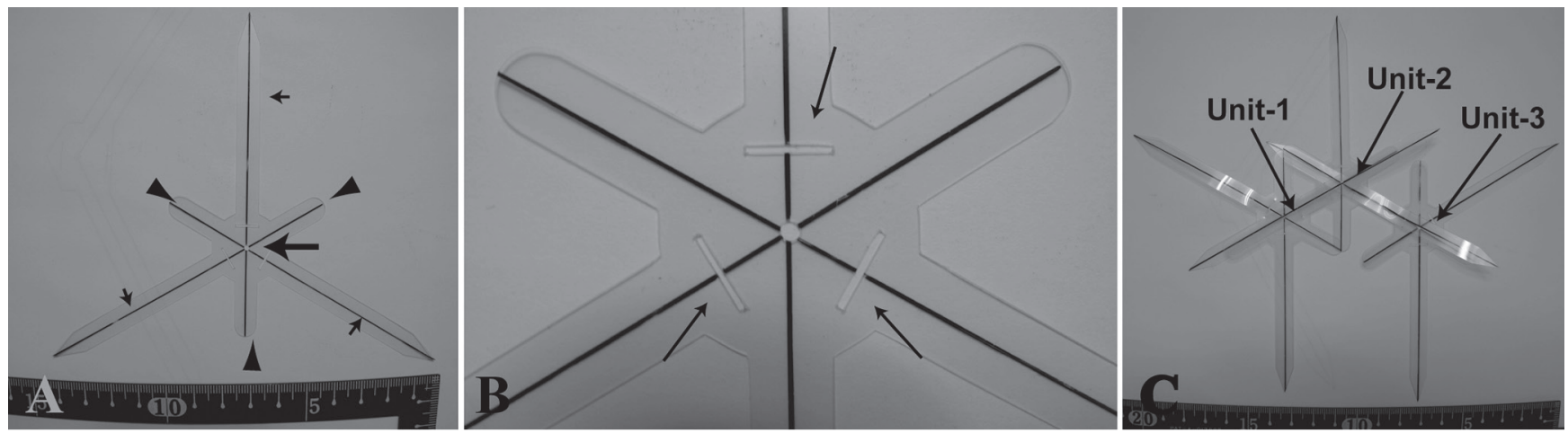

Fig. 2 MST template. A: An SU comprising the MST template is shown. It has three wings indicating three branches (small arrow) radiating from the SP at the center (large arrow) and three tabs for convenient movement on the counterpart of the branch (arrowhead). B: Extended image of an SU. An SU has three slits (arrow) near the center, through which a wing of another SU can be passed through. C: An MST template for a pentagonal dural window is shown. This template was assembled by combining three SUs (units 1,2 and 3 ) connected to each other's wings through slits. 


\section{Results}

\section{Geometrical analysis}

According to the geometrical analysis, the shortest incision design for a pentagon consists of three interconnected SPs and their seven branches (Fig. 1, right).

Brief proof: Suppose A1, A2 and P are to be interconnected with line segments. We can describe every pattern of this interconnection by placing a TPI (Q) interconnecting each vertex anywhere on the same plane (Fig. 3A). For the understanding auxiliary, consider two equilateral triangles, $\triangle \mathrm{A} 1 \mathrm{~B} 1 \mathrm{~A} 2$ and $\triangle \mathrm{A} 1 \mathrm{Q}$ 'Q. If we plot $\triangle \mathrm{A} 1 \mathrm{~B} 1 \mathrm{~A} 2$ and $\triangle \mathrm{A} 1 \mathrm{Q}$ 'Q, as shown in Fig. $3 \mathrm{~B}$, the total length of the line segments interconnecting the three points A1, A2 and $P$ (the sum of line segments $A 1 Q, A 2 Q$ and $P Q)$ is equal to the path $B 1 Q^{\prime} Q P$ because $\triangle A 1 A 2 Q$ and $\triangle \mathrm{A} 1 \mathrm{~B} 1 \mathrm{Q}$ ' are congruent. Therefore, the $\mathrm{Q}$ that results in the shortest path B1Q'QP is the location of the TPI interconnecting $\mathrm{A} 1, \mathrm{~A} 2$ and $\mathrm{P}$ with the shortest total length. Path B1Q'QP is the shortest when Q' and Q are on line B1P (Fig. 3C); then, the sum of the connecting line with $\mathrm{A} 1, \mathrm{~A} 2$ and $\mathrm{P}$ is the shortest in the design described in Fig. 3D. In other words, the total length of the shortest set of line segments interconnecting the three given points $\mathrm{A} 1, \mathrm{~A} 2$ and $\mathrm{P}$ is the distance between $\mathrm{B} 1$ and $\mathrm{P}$. Moreover, the TPI connecting these three points is located at the intersection of lines B1P, B2A2 and $\mathrm{B} 3 \mathrm{~A} 1$ if we plot equilateral triangles $\triangle \mathrm{B} 2 \mathrm{~A} 1 \mathrm{P}$ and $\triangle \mathrm{B} 3 \mathrm{PA} 2$, and the angle between any pair of branches around the TPI is $2 / 3 \pi$. The TPI has a property of an SP, and the network including these branches is called the MST of $\triangle \mathrm{A} 1 \mathrm{~A} 2 \mathrm{P}$ (Fig. 3D). Hence, we can consider point B1 (the outer vertex of the

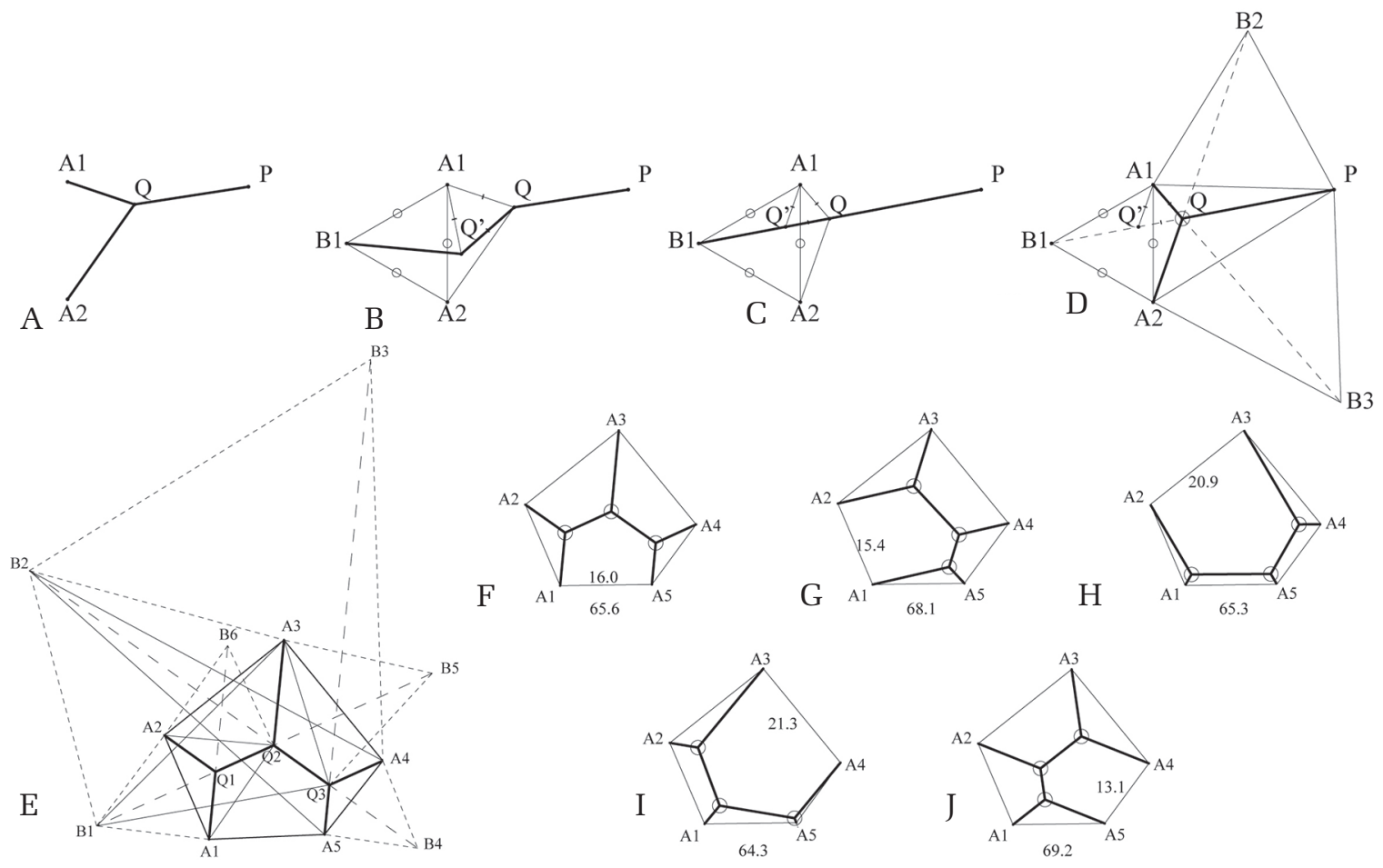

Fig. 3 Proofs for the shortest network. The theory is developed in the text. A: Consider three points, A1, A2 and P, interconnected with a trifurcated line segment (thick line). Q indicates the TPI of the segment. B: Equilateral triangles $\triangle \mathrm{B} 1 \mathrm{~A} 2 \mathrm{~A} 1$ and $\triangle \mathrm{A} 1 \mathrm{Q}$ 'Q (fine line) are given for auxiliary understanding. Small circles or small lines marked on line segments indicate equal lengths. Path B1Q'QP is drawn with thick line. C: Path B1Q'QP (thick line) is the shortest when $Q^{\prime}$ and $Q$ are on line segment B1P. D: The shortest design interconnecting A1, A2 and P, meaning the MST of $\triangle \mathrm{A} 1 \mathrm{~A} 2 \mathrm{P}$, is shown (thick lines). Equilateral triangles $\triangle \mathrm{B} 2 \mathrm{~A} 1 \mathrm{P}$ and $\triangle \mathrm{B} 3 \mathrm{PA} 2$ (fine line) are given. $\mathrm{Q}$ as SP (surrounded by circle) is on line segments B1P, B2A2 and B3A1 (broken line). E: Construction of the MST using Melzak's algorithm. The Steiner tree (thick line) of the pentagon A1A2, namely, A5 (thin line), is drawn. Equilateral triangles $\triangle \mathrm{B} 1 \mathrm{~A} 1 \mathrm{~A} 2, \triangle \mathrm{B} 2 \mathrm{~B} 1 \mathrm{A3}, \triangle \mathrm{B} 3 \mathrm{~B} 2 \mathrm{~A} 4, \triangle \mathrm{B} 4 \mathrm{~A} 4 \mathrm{~A} 5, \triangle \mathrm{B} 5 \mathrm{~A} 3 \mathrm{Q} 3$ and $\triangle \mathrm{B} 6 \mathrm{~A} 2 \mathrm{Q} 2$ (detailed broken line) are given for auxiliary understanding. Rough broken lines are drawn to identify the SP of the pentagon. Q1, Q2 and Q3 are the SPs of the Steiner tree. F, G, H, I and J: Five Steiner trees derived from a given pentagon are shown (thick line). SPs are surrounded by circles. The total length of each tree is indicated below the pentagon. The length of the side that is enclosed by start and end points of Melzak's algorithm is indicated at the side of each pentagon. 
plotted triangle) as an alternative to points A1 and A2 (two vertices at the bottom of the plotted triangle) when they have the shortest distance to any P. If we try to determine the shortest network of five vertices of a given pentagon A1A2, namely, A5 (Fig. 3E), constructing B1 at a vertex of triangle B1A1A2 allows us to consider B1 as an alternative point to A1 and A2 connecting the shortest with any point in the pentagon. In the same manner, if we construct B2, B3 and B4 as alternative points to B1 and A3, B2 and A4, A4 and A5, respectively, the SP of $\triangle \mathrm{B} 2 \mathrm{~A} 5 \mathrm{~A} 4$ comprises one of the TPIs of the shortest network of the pentagon because we can consider B2 as an alternative point to $\mathrm{A} 3$ and $\mathrm{B} 1$ (which is an alternative to A1 and A2). As mentioned above, the SP of $\triangle \mathrm{B} 2 \mathrm{~A} 5 \mathrm{~A} 4$ is at the intersection of lines B2B4 and B3A5, named Q3. We then consider the shortest network of $\triangle \mathrm{Q} 3 \mathrm{~A} 3 \mathrm{~B} 1$. We construct $\mathrm{B} 5$ at a vertex of triangle $\triangle \mathrm{B} 5 \mathrm{~A} 3 \mathrm{Q} 3$; then, the SP of $\triangle \mathrm{Q} 3 \mathrm{~A} 3 \mathrm{~B} 1$ is at the intersection of line B5B1 and B2Q3, named Q2. Finally, we consider the shortest network of $\triangle \mathrm{Q} 2 \mathrm{~A} 2 \mathrm{~A} 1$. We construct B6 at a vertex of triangle $\triangle \mathrm{B} 6 \mathrm{~A} 2 \mathrm{Q} 2$; then, the $\mathrm{SP}$ of $\triangle \mathrm{Q} 2 \mathrm{~A} 2 \mathrm{~A} 1$ is at the intersection of lines B6A1 and B1Q2, named Q1. The network consists of three SPs-Q1, Q2, Q3and seven branches of these SPs form the shortest network (Steiner tree) connecting five vertices of the pentagon. Such a solution is called Melzak's algorithm. ${ }^{2}{ }^{2}$ If we apply this algorithm to the same pentagon with other starting points from A2, A3, ...A5, we obtain four other types of Steiner tree (Figs. 3F-3J). We compared the lengths of these five trees, and the shortest is shown in Fig. 3I. Several trials conducted to compare the total lengths of the Steiner trees of several given pentagons revealed that the shortest tree was constructed using Melzak's algorithm by starting from the end of the longest side of the pentagon. We could not demonstrate this result geometrically, but experimental trials confirmed its legitimacy.

Here, we solved the problem by applying Melzak's algorithm; however, this algorithm cannot be applied for every type of pentagon. If fully applicable, the vertices of such pentagons have no obtuse angles greater than $2 / 3 \pi{ }^{3)}$ In the cases, such as the pentagon depicted in Fig. 4, Melzak's algorithm cannot be completely applied starting from an end of the longest side and always has a wide-angle vertex greater than $2 / 3 \pi$. Identifying the shortest design in these pentagons is complicated, but such uneven pentagons are rarely encountered.

\section{Usage of MST template in clinical practice}

After a craniotomy is performed, all the vertices of a quadrilateral or pentagon to be opened must be marked on the exposed dural surface before the incision is made (Fig. 5A). To create the shortest incision design for a quadrilateral dural window, two SUs can be assembled to create an MST template, and the distance between their SPs can be adjusted to align the four radiating wings on the four vertices of the quadrilateral. Consequently, the combination of their branches shows us the shortest incision design. When a pentagonal window must be created, three SUs can be assembled to create an MST template (Figs. 2C and 5B). Using this approach, we can create five candidates for the shortest incision design. As noted above, the shortest design encloses both ends of the longest side of the pentagon within each wing of the SUs at both ends of the combination. Then, we can mark the selected design on the dura mater (Fig. 5C). Using the MST template, we could create the shortest design within a minute in all clinical cases ( 7 cases).

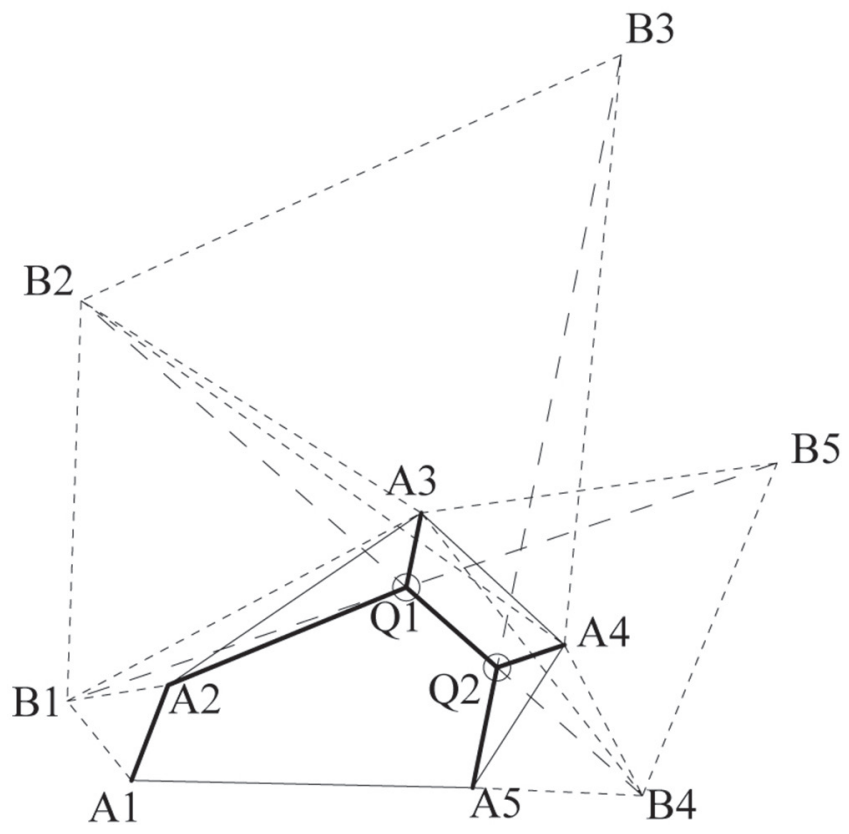

Fig. 4 A case in which the shortest network does not have three SPs. An MST (thick line) of the pentagon A1A2, namely, A5 (thin line), with a wide angle at A2 $(>2 / 3 \pi)$ is described. Equilateral triangles $\Delta \mathrm{B} 1 \mathrm{~A} 1 \mathrm{~A} 2$, $\triangle B 2 B 1 A 3, \triangle B 3 B 2 A 4, \triangle B 4 A 4 A 5$ and $\triangle B 5 A 3 B 4$ (detailed broken line) are given for auxiliary understanding. Rough broken lines are drawn to identify the SP of the Steiner tree. We defined two SPs (Q1 and Q2) and their branches by applying Melzak's algorithm but could not define Q3 as the remaining SP using the algorithm. We could not set the SP interior to $\triangle \mathrm{A} 2 \mathrm{~A} 1 \mathrm{Q} 1$ because $\angle Q 1 A 2 A 1$ is greater than $2 / 3 \pi$. The shortest pattern connecting A2, A1, and Q1 is path A1A2Q1. Each angle for the circular arcs in these drawings is equal to $2 / 3 \pi$. 


\section{Comparison to the conventional method}

The conventional dural incision used to open a pentagonal window involves cutting first in a semicircular shape and then along three auxiliary lines, creating a polyline along with the four sides of the pentagonal window or a radiation line for five vertices. However, the total cutting length of these standard designs varies and is not the shortest possible length. In the case of Fig. 5 (the pentagon has sides of $12,10.2,11.2,9.4$, and $10.3 \mathrm{~cm}$ in length), the shortest design requires $40.4 \mathrm{~cm}$ of dural incision (Fig. 4C), whereas the conventional method using a semicircle and three auxiliary lines, a polyline or radiation lines requires $42.9,41.4$, or $45.4 \mathrm{~cm}$, respectively (Figs. 4D-4F).

\section{Discussion}

First, we determined that the shortest incision design for pentagonal dural window is a Steiner tree composed of three interconnected SPs. Second, we created the MST template that simply shows us the shortest incision design for both quadrilateral and pentagonal dural windows.

We presented the shortest incision design for a pentagonal dural window as a Steiner tree. Our method above can be adopted for pentagons to which Melzak's algorithm can be applied, starting from the end of the longest side. Herein, we discuss the geometry of the shortest incision design. To our knowledge, this is the first study to discuss the geometric design of a dural incision for a pentagonal dural window.

The MST template makes it easy to determine the shortest incision design for a polygonal dural window. The template is made of a clear polypropylene film. The properties of this material allow us to easily bend the template in a vertical direction along with the curve of the brain surface, but it does not bend on the horizontal plane intraoperatively. Accordingly, the template maps an accurate shortest incision design.

This new method has a few problems in practice. One is that the proximity to the dural venous sinuses and the location of the underlying pathology might restrict the use of our incision design. A second problem is
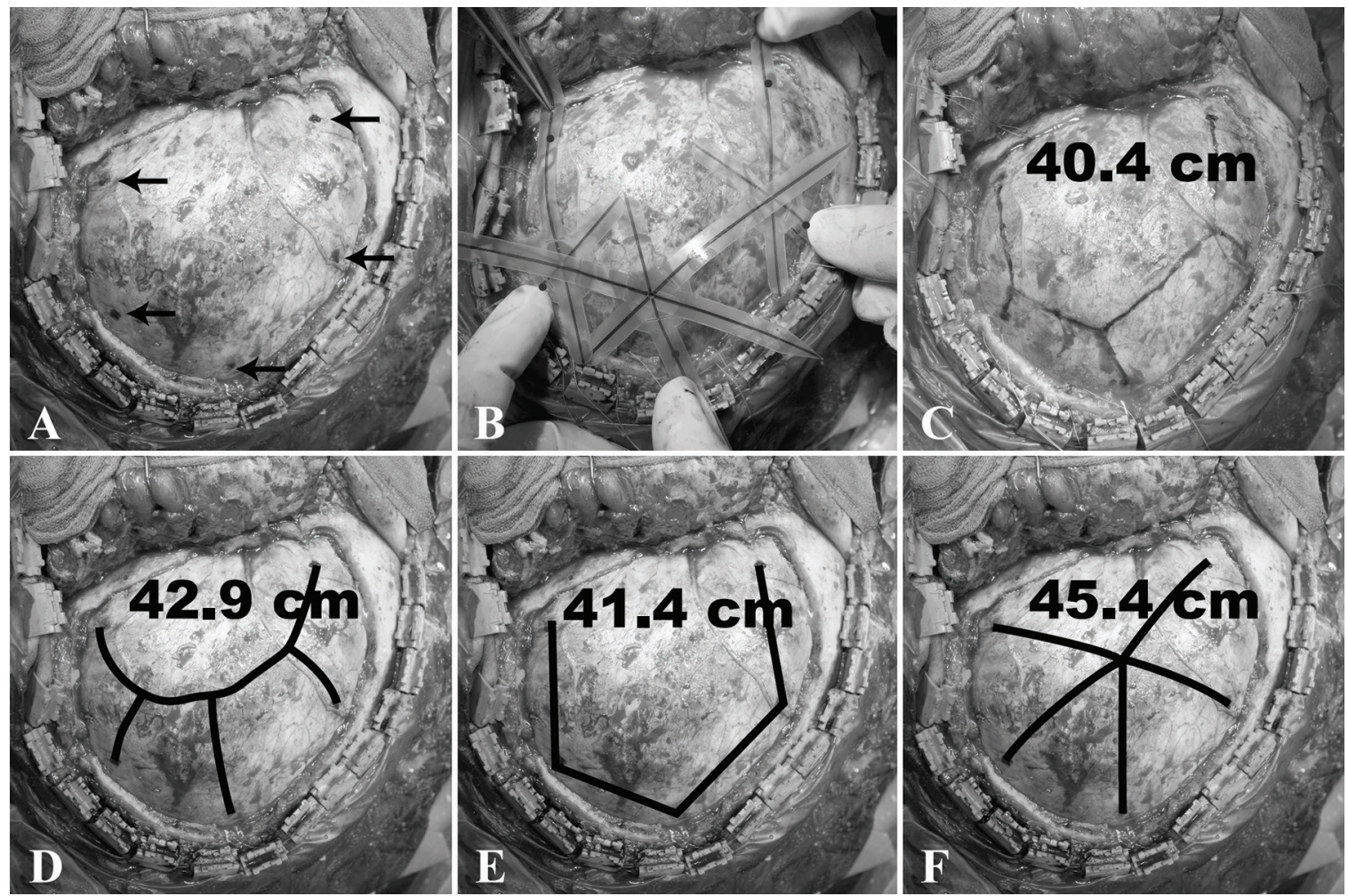

Fig. 5 Intraoperative usage of the MST template. A, B and C: The intraoperative use of an MST template is shown. A: First, the vertices (arrow) of a sufficiently wide polygon (pentagon here) are marked. B: Then, the assembled MST template is applied to the dura mater with five wings meeting on each mark. Both ends of the longest side of the pentagon must be enclosed by the wings of SUs of the assembled MST template. C: The MST of this pentagon is marked on the dura mater, creating the shortest incision design. D, E and F: Incision designs of a semicircle and auxiliary lines (D), a polyline (E) and radiation lines (F) are drawn using a thick line on the same craniotomy picture. C, D, E and F: The total incision lengths are shown in the picture. 


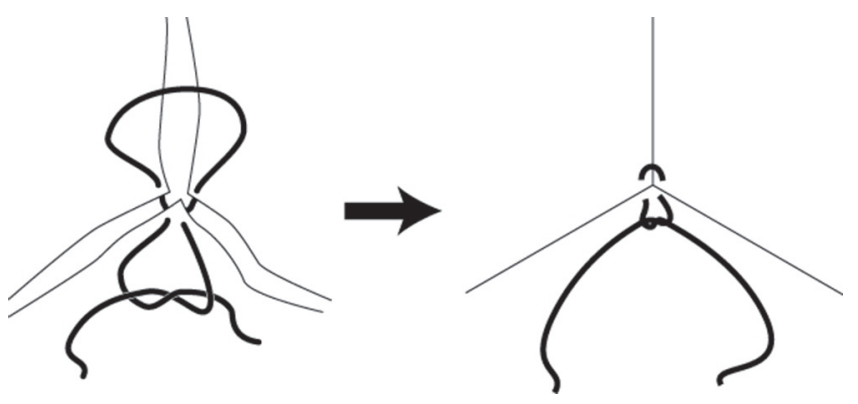

Fig. 6 TPI closure. The closure of the tripartite dural incisions at the TPI is indicated. A purse string suture with four stitches is sufficiently simple for tight closures. The cutting line (thin curved line) and suture thread (thick curved line) are indicated.

that surgeons may feel that it is more difficult to close tripartite dural incisions at the TPI of this design. We usually close TPI of the incision line by a simple purse string suture with four stitches (Fig. 6). Nonetheless, the approximately 1-5 $\mathrm{cm}$ shorter incision lengths created using this method will relieve neurosurgeons from cumbersome dural closures. In this paper, we first geometrically examined the shortest incision design for a pentagonal dural window, and we then introduced an MST template that allows the easy intraoperative creation of the shortest incision design.

\section{Conflicts of Interest Disclosure}

The authors report no conflicts of interest concerning the materials or methods used in this study or the findings specified in this paper. All authors who are members of The Japan Neurosurgical Society (JNS) have registered online Self-reported COI Disclosure Statement Forms through the website for JNS members.

\section{References}

1) Nagai M, Yamamoto $T$, Oguma $H$, Watanabe E: Planar geometrical analysis for design of the shortest incision to open the dura mater: technical note. Neurol Med Chir (Tokyo) 53: 61-64, 2013

2) Melzak ZA: On the problem of Steiner. Canadian Mathematical Bulletin 4: 143-148, 1961

3) Gilbert EN, Pollak HO: Steiner minimal trees. SIAM J Appl Math 16: 1-29, 1968

Address correspondence to: Mutsumi Nagai, MD, PhD, Department of Neurosurgery, Saitama Medical Center Jichi Medical University, 1-847 Amanumacho, Omiya-ku, Saitama, Saitama 330-8503, Japan. e-mail: mnagai@jichi.ac.jp 